Several prospective studies have demonstrated that aPL are predictive of vascular events in patients with SLE. Though conflicting results exist, we have not noted positive associations between $\mathrm{aPL}$ and accelerated atherosclerosis in SLE, rather we believe that it is the pro-thrombotic state, which is the major cause of vascular events in the aPL positive SLE subgroup. Microvascular disease is an often unrecognised and difficult to diagnose feature in the aPL positive SLE subgroup. We have noted that up to $15 \%$ of patients diagnosed with nephritis have microvascular pathology in accordance with APS nephropathy in renal biopsies.

The aPL positive SLE subgroup has also been demonstrated to have a more rapid accrual of damage, where in addition to vascular damage neuropsychiatric damage makes a notable contribution.

When investigating primary APS patients, we and others have noted traits that are normally seen and attributed to SLE, such as low platelets, complement consumption, a low grade of systemic inflammation and even low titers of anti-DNA antibodies. Thus, the distinction between these two groups, APS secondary to SLE and primary APS, is not always clear.

Disclosure of Interest: None declared

DOI: 10.1136/annrheumdis-2018-eular.7817

\section{SP0166 ALL YOU NEED TO KNOW ABOUT KIDNEY DISEASE IN ANTIPHOSPHOLIPID SYNDROME}

M. Tektonidou. First Department of Propaedeutic and Internal Medicine, Medical School, National and Kapodistrian University of Athens, Athens, Greece

Antiphospholipid syndrome (APS) can affect any vascular bed and is characterised by a plethora of clinical manifestations related with different organ systems involvement. Accordingly, APS can affect any part of kidney vasculature and parenchyma such as renal arteries and veins, intra-renal arteries and arterioles, and glomerular capillaries. APS-associated nephropathy was first described in patients with primary APS, characterised by acute thrombotic lesions in glomeruli and/or arterioles (thrombotic microangiopathy) and chronic vascular lesions such as fibrous intimal hyperplasia of arterioles and interlobular arteries, organised thrombi with or without recanalisation, and fibrous arterial and arteriolar occlusions or focal cortical atrophy. APS nephropathy lesions have also been later described in patients with SLE-associated APS and SLE patients with positive antiphospholipid antibodies but without APS, independently of lupus nephritis. ${ }^{2,}$ The most common clinical manifestations of APS nephropathy include hypertension, microscopic hematuria, proteinuria (from mild to nephrotic range), and a usually mild renal insufficiency. Arterial thromboses (especially stroke), pulmonary embolism, livedo reticularis, anticardiolipin antibodies, and lupus anticoagulant were strongly associated with histologic lesions of APS nephropathy. During the follow-up period, manifestations of APS (especially arterial thromboses) developed more frequently in SLE/non-APS patients with APS nephropathy than in those without APS nephropathy lesions. In the Sydney classification criteria for APS, APS nephropathy has been included in non-criteria APS manifestations. The significant association between the presence of APS nephropathy and antiphospholipid antibodies suggests a pathogenetic role of antiphospholipid antibodies in the development of this nephropathy. Data from experimental and clinical studies support also a potential role of complement cascade activation, tissue factor activation, and activation of mTORC in APS nephropathy pathogenesis. Currently, there is no consensus on the treatment of APS nephropathy. Updated evidence about the role of anticoagulation, hydroxychloroquine, statins, and targeted therapies such as B-cell directed therapies, complement inhibition, tissue factor inhibition, and mTOR pathway inhibition will be discussed.

\section{REFERENCES:}

[1] Nochy D, Daugas E, Droz D, et al. The intrarenal vascular lesions associated with primary antiphospholipid syndrome. J Am Soc Nephrol 1999;10:507-518

[2] Daugas E, Nochy D, Huong du LT, et al. Antiphospholipid syndrome nephropathy in systemic lupus erythematosus. J Am Soc Nephrol 2002;13:42-52.

[3] Tektonidou MG, Sotsiou F, Nakopoulou L, et al. Antiphospholipid syndrome nephropathy in patients with systemic lupus erythematosus and antiphospholipid antibodies: prevalence, clinical associations, and longterm outcome. Arthritis Rheum 2004;50:2569-2579.

[4] Miyakis S, Lockshin MD, Atsumi T, Branch DW, Brey RL, Cervera R, Derksen RH, DE Groot PG, Koike T, Meroni PL, Reber G, Shoenfeld Y, Tincani A, Vlachoyiannopoulos PG, Krilis SA. International consensus statement on an update of the classification criteria for definite antiphospholipid syndrome (APS). J Thromb Haemost 2006;4:295-306.

Disclosure of Interest: None declared

DOI: 10.1136/annrheumdis-2018-eular.7833
SATURDAY, 16 JUNE 2018

The links between gout and kidney function

\section{SP0167 RENAL URATE TRANSPORTERS (SUMMARY FOR} CLINICIANS)

F. Perez Ruiz. Department of Medicine, University of the Basque Country, Baracaldo, Spain

Gout was, and still is in some academic environments, a "metabolic" disease. The advent of allopurinol in the mid of the XXth century, the first xanthine-oxidase inhibitor, precluded that most subjects with "primary gout" were to suffer from overproduction of uric acid. No actual overproduction was neatly demonstrated and some empiric observations showed that "renal underexcretion" was working in most patients with gout.

In 2002, Enomoto and coworkers characterised the first renal urate transporter, URAT1, encoded by SL22A12, showing that the human knockout for URAT1 was associated with familiar hypouricemia. A bunch of uric acid transporters have been characterised since then, polymorphisms of some of them being associated with variability in renal handling of urate. The function of ransporters is complex: PDZK1 (also known as NHERF3) is a scaffolding protein that binds to several urate transporters such as URAT1, OAT4, and NPT1. Therefore, PDZK1 plays a pivotal role in forming a urate-transporting multimolecular complex (also named "urate transportsome") in humans. Hyperuricemia is no more a metabolic disease: it is a "transportpathy". In addition, linkage of urate transporters to $\mathrm{Na}, \mathrm{P}$, and sugars may help understanding some comorbid conditions associated to hyperuricemia and gout, such as diabetes and hypertension.

In addition to the previously referred, ABCG2 is a cassette binding protein expressed in the in the kidney and more importantly in the intestine, where is involved with active excretion. The discovery of ABCG2 helped to explain that apparent overproduction in some patients is a "renal overload" due to impaired intestinal excretion.

A summarised knowledge on the urate transporters may be useful for clinicians implicated in the management of gout, as it may explain why XOls efficacy does differ in patients with apparent overproduction, why allopurinol response may be blunted in some patients, how targeting transporters may be helpful for the development of new urate-lowering molecules, and how to explain efficacy and safety models for uricosurics and combination therapies.

Disclosure of Interest: F. Perez Ruiz Grant/research support from: Asociación de reumatologos de Cruces, Consultant for: Grünenthal, Menarini, Speakers bureau: Grünenthal, Menarini

DOI: 10.1136/annrheumdis-2018-eular.7820

\section{SP0168 THE USE OF XO INHIBITORS IN CKD - PROS AND CONS}

N. Dalbeth. University of Auckland, Auckland, New Zealand

The association of chronic kidney disease (CKD) and hyperuricaemia is well established, and many observational studies have reported that hyperuricaemia is associated with development and progression of CKD. Potential mechanisms of this observation will be discussed, including the potential for urate as an 'innocent bystander' or as a causal mediator contributing directly to kidney injury. Xanthine oxidase inhibitors (XOls) are the most widely used urate-lowering drugs Current evidence for efficacy and safety of XOls for preventing or delaying progression of chronic kidney disease will be presented, both in the general population and in people with gout. The challenges of $\mathrm{XOI}$ use in people with CKD will also be discussed, with specific reference to allopurinol dosing in CKD, and cardiovascular safety of febuxostat.

Disclosure of Interest: N. Dalbeth Consultant for: Kowa, Horizon

DOI: 10.1136/annrheumdis-2018-eular.7766

SATURDAY, 16 JUNE 2018

Big data in pre-clinical research

\section{SP0169 INTEGRATION OF OMICS DATA FOR PREDICTING RESPONSE TO ANTI TNF TREATMENT}

L. Padyukov. Rheumatology Unit, Department of Medicine Solna, Karolinska Institutet, Stockholm, Sweden

Successful development of biologics for targeting specific molecules, like TNF was a hallmark of new era in therapy of inflammatory and rheumatic diseases. 